\title{
Die Chinaalkaloïde und Sulfocyankalium.
}

\author{
Von F. Schrage in Pewsum.
}

Im Archiv der Pharmacie von 1865. Bd. 124, S. 143 wurden Arbeiten Stoddart's mitgetheilt, welche u. A. ergaben, dass die in dort vorgeschriebener Weise bewirkten Niedersehläge der Chinasalze mit Rhodankalium bei einiger Uebung mittelst einer Loupe sofort zu unterscheiden sind: Chinin bildet nach Stoddart dünne lange Nadeln, Chinidin runde krystallinische Gruppen, Cinchonin grosse gut geformte Prismen.

Mit Untersuchungen ähnlicher Art beschäftigt, habe ich eine Reihe vergleichender mikroskopischer Prüfungen gemacht, welche bestimmtere, im Archiv der Pharmacie 1874. Heft 6, S. 504 veröffentliche Resultate gaben, die ich auch heute noch als der Hauptsache nach richtig bezeichnen muss.

Eigene Erfahrungen und die Mittheilungen Anderer haben mich überzeugt, dass es für das Verständniss dieser subsidiär sehr brauchbaren Reaction wünschenswerth ist, die Bedingungen ihres Eintretens näher zu präcisiren, und dass es nöthig ist, das Cinchonidin in den Kreis der Beobachtungen aufunehmen, weil dieses in neuerer Zeit in der Fabrikation, im Handel und im Verbrauch eine grössere Bedeutung erhalten hat.

Es beziehen sich demnach die nachfolgenden Mittheilungen auf die 4 im Handel vorkommenden Alkaloïde Chinin, Cinchonin, Cinchonidin und Chinidin.

Unter letzterem verstelie ich dasjenige Alkalö̈d, welches Kerner $\beta$ Chinidin, Hesse Conchinin nennt, und halte an der Benennung Chinidin fest, weil Kerner's in Fresenius' Zeitschrift fiir analytische Chemie 1862. Heft 2 veröffentlichte Untersuchungsmethode für das Chininsulfat durch Aufnahme in die Pharmacopoea germanica legalisirt ist, weil die Pharmacopoea austriaca und ihr entsprechend Hirsch in seiner Prüfung der Arzneimittel von 1875 ebensowohl, wie a. a. O. Hager und Mohr die Kerner'sche Bezeichnung ihren Arbeiten zu Grunde legen.

Die Versuche erstreckten sich auf die Verbindungen des Chinins mit Schwefelsäure, Chlorwasserstoffsäure, Essigsäure, 
Salicylsäure, Salpetersäure und Baldriansäure, ferner auf die Chlorate und Sulfate des Cinchonins, Chinidins und Cinchonidins.

An käuflichem Chinidin kamen zur Untersuchung Producte aus den Fabriken von C. F. Böhringer und Söhne in Mannheim, Fr. Jobst in Stuttgart, C. Zimmer in Frankfurt und Böhringer und Geyer in Stuttgart.

Bringt man neutrale Salzlösungen der 4 genannten Chinaalkaloïde im Reagircylinder mit Rhodankaliumlösung zusammen, so entstehen auch noch bei sehr grosser Verdünnung weisse Niederschläge.

Die Niederschläge des Rhodankaliums mit Chinin und Cinchonidin sind leicht und vollständig löslich ebensowohl im Ueberschuss der zugesetzten Chinin- resp. Cinchonidinlösung, als auch im Ueberschuss von Rhodankalium; nicht oder doch nur wenig löslich im Ueberschuss des einen oder des andern Agens sind die Rhodanniederschläge von Chinidin und Cinchonin.

Setzt man zu einer kalt gesättigten Lösung von Chininsulfat oder zu einer in dem Verhältniss von 1:100 bereiteten Lösung von Cinchonidinsulfat einen Tropfen concentrirter Lösung von Rhodankalium (1:1), so wird der za Boden sinkende Tropfen dort einen Niederschlag erzeugen, welcher bei mässiger Bewegung des Reagirglases sich wieder löst. Aus dieser Lösung scheiden sich nach einiger Zeit sternförmig gruppirte Nadeln aus, und eine wenig modificirte sternförmige Ausscheidung tritt ebenfalls ein, wenn man den entstandenen Niederschlag durch Uebermaass von Rhodankalium zur Lösung bringt und einige Zeit ruhig bei Seite setzt. Die Zeit des Eintretens dieser Reaction hängt wesentlich ab von der relativen Menge der in Wechselwirkung tretenden Flüssigkeiten und es ist leicht, dieses Verhältniss anschaulich zu machen, wenn man eine bei $15^{\circ}$ gesättigte Lösung von Chininsulfat in der Weise in zehn Reagirgläser vertheilt, dass das erste Glas 1 C.C., das nächste 2 C.C., und jedes folgende 1 C.C. mehr, bis das letzte Glas 10 C.C. enthält, alsdann in jedes Glas einen Tropfen Rhodankaliumlösung (1:1) fallen lässt 
und durch Umschwenken den entstandenen Niederschlag wieder löst. Nach diesem Verfahren gab in mehreren Versuchen Chininsulfatlösung:

1 C.C. keine klare Lösung,

2 und 3 C.C. opalisirende Lösungen,

4 und mehr C.C. klare Lösungen.

1 C.C. gab nach 2 Minuten schon erheblichen krystallinischen Niederschlag,

2 C.C. nach 5 Minuten dasselbe,

3 C.C. nach 5 Minuten schwachen Niederschlag, nach längerer Zeit Ausscheidung zahlreicher, sternförmig gruppirter Nadeln,

4 C. C. ähnlich wie 3 C.C.,

5 und 6 C.C. schön ausgebildete mattglänzende Sterne,

7 C. C. Ausscheidungen erst nach $1-2$ Stunden, Sterne erst nach 10 Stunden,

8, 9 und 10 C.C. bei einigen Versuchen nach 10 Stunden schwache krystallinische Ausscheidungen, bei andern nichts.

Die bei Wiederholung der Versuche stets vorkommenden Schwankungen dürften auf der Unmöglichkeit beruhen, Tropfen von absolut gleicher Grösse zu schaffen.

Aehnliche Erscheinungen beobachtet man, wenn man statt Chininsulfatlösung eine Auflösung von Cinchonidinsulfat im Verhältniss von 1:100 verwendet.

Wenn man behufs der mikroskopischen Prüfung die Lösungen der neutralen Chinaalkalö̈dsalze in der Weise mit Rhodankalium zusammenbringt, dass man auf einem Objectträger einen Tropfen der Salzlösung und einen Tropfen Rhodankaliumlösung $(1: 1)$ nebeneinander bringt, ohne dass sie sich berühren, durch das Auflegen eines dünnen Deckgläschens aber zum Zusammenfliessen veranlasst werden, so entsteht an der Berührungsfläche eine weisse Ausscheidung, deren Beschaffenheit von der Natur der mit dem Alkaloïd verbundenen Säure nicht abhängt.

Man kann, wie früher bereits angegeben wurde, die Salze der Chinabasen einige Zeit mit destillirtem Wasser 
zusammenbringen, und die so erhaltene filtrirte gesättigte Lösung zur mikroskopischen Prüfuilg verwenden, allein in den Fällen, wo es sich um eine Vergleichung der Beobachtungsresultate handelt, empfiehlt es sich, alle hinreichend löslichen Salze in dem Verhältnisse von 1: 100 gelöst zu verwenden, Rhodankalium stets in gleichen Theilen Wasser gelöst.

Bei schwerer löslichen Salzen, z. B. bei neutralem Chininsulfat ist eine kleine Steigerung der Lösungstemperatur nützlich, aber sicherer zum Ziele führend, wenn man das Sulfat durch Zusatz von $25 \%$ Chlorbaryum in Chlorat überfuihrt.

Abgesehen von der Löslichkeitsïnderung ist nicht bemerkbar, dass die Temperatur einen wesentlichen Einfluss auf das Eintreten der charakteristischen Erscheinungen hat.

Die Grösse der zur Verwendung kommenden Tropfen ist um so mehr von Bedeutung, weil es sich bei der mikroskopischen Untersuchung nicht ausschliesslich um die Form der gebildeten Krystalle, amorphen oder halb amorphen Gebilde handelt, sondern sehr wesentlich auch um die Gruppirung dieser verschiedenen Gebilde. Abgesehen von einzelnen, bei vergleichenden Arbeiten sich von selbst ergebenden Fällen ist es zweckmässig, mittelst eines Glasstäbchens die Flüssigkeiten auf den Objectträger zu bringen in der Weise, dass von beiden Flüssigkeiten nur etwa je $1 / 3$ bis $1 / 4$ eines fallenden Tropfens zur Verwendung kommt, und dann das möglichst horizontal liegende Object nach etwa halbstïndiger absoluter Ruhe bei etwa 110 facher Vergrösserung beobachtet wird.

Der Niederschlag, welcher an der Berührungsfäche der beiden Flüssigkeiten entsteht, stellt sich selbst bei 500 facher Vergrösserung noch als feinen amorphen Staub dar.

Wenn nicht bei zu grosser Menge dieses Niederschlages die Beobachtung unklar wird, also bei Verwendung nicht zu concentrirter Chinasalzlösung, dann gelingt es der Regel nach leicht zu beobachten, dass diese staubförmige, sei es flüssige, oder nicht flüssige Ausscheidung sich zu dickeren Tropfen sammelt, welche theils ohne weiteres krystallinisch 
werden, theils durch Auflösung wieder verschwinden und so das Material zum Wachsthum der entstandenen krystallinischen Gebilde liefern. Im Allgemeinen geht diese Umwandlung bei allen Chinasalzen um so schneller vor sich, je concentrirter die verwendeten Lösungen sind. Bei Lösungen von 1:100 beginnt sie schon in der ersten Minute, ist nach 5 Minuten schon erheblich entwickelt und nach 30 Minuten der Regel nach beendet.

Nicht selten werden sich nachträglich noch weitere Entwickelungen zeigen und in sofern empfiehlt sich eine wiederholte Besichtigung auch noch nach mehreren Stunden, und zwar insbesondere dann, wenn man mit Cinchonidinsalzen zu thun hat.

Abweichungen von der Regel erklären sich daraus, dass schon aus rein mechanischen Gründen nicht immer genau gleich grosse Mengen der beiden Flüssigkeiten in Wechselwirkung treten, und, wie die Resultate der Reagircylinderversuche zeigen, das relative Mengenverhältniss für diese sehr oft durch Auflösung und Wiederausscheidung in der Ruhe bedingten Erscheinungen sowohl im Allgemeinen als auch insbesondere für ihre Eintrittszeit von durchschlagender Bedeutung ist.

Das längere Verharren der Ausscheidungen im flüssigen Zustande hat regelmässig die Bildung sehr grosser Tropfen zur Folge.

Zu ihrer Umbildung in Krystalle scheint die sie umgebende Flüssigkeit wesentlich erforderlich und deren Verschwinden durch Verdunstung der Grund des vorkommenden endgültigen Verharrens der Tropfen in amorphem oder halbkrystallinisch entwickeltem Zustande zu sein.

Die entstehenden Gebilde vereinigen sich zu mehr oder weniger zusammengesetzten Figuren, deren Form für die einzelnen Chinasalze und für Gemische derselben charakteristisch ist und theilweise nicht gut anders beschrieben werden kann, als durch Vergleich mit verschiedenen Pflanzen.

Allgemein unterscheiden sich die entstehenden Gruppirungen durch ihre Wachsthumsrichtung, insofern diese 
entweder einseitswendig, oder nach allen Seiten hin gewendet ist.

Da alle nicht isolirt bleibenden Krystalle oder halbamorphen Bildungen darin übereinstimmen, dass sie zunächst von einem Punkte ausgehen und dann unter kleinem Winkel divergiren, so ist die Grundform der einseitswendigen $\mathrm{Ge}$ bilde der Fächer, die Grundform den allseitswachsenden Gebilde der Stern.

Mit dem Wachsthum der Strahlen der combinirten krystallinischen Figuren geht ein mit dem Maasse dieses Wachsthums Schritt haltendes Verschwinden der die Krystalle umgebenden Tropfen vor sich, welches sich durch zunehmende Melligkeit des Gesichtsfeldes ankündigt.

Je dicker die Tropfenschicht ist, um so mehr verästelt sich der Strahl; je dünner sie ist, um so einfacher bleibt der Strahl. Ist die umgebende Tropfenschicht in verschiedenen Richtungen ungleich dicht, so sind auch diesem Verhältnisse entsprechend die Strahlen ungleich complicirt; ist die Tropfenschicht nach einer Seite hin weniger ausgedehnt, so entstehen an dieser Seite auch in dem Maasse weniger lange Strahlen.

Die sternförmige Gruppirung ist charakteristisch für die Salze von

\section{Chinin und Cinchonidin.}

Der Uebergang der amorphen Tropfen in die Sternform vollzieht sich schnell und vollständig beim Chinin, insoweit nicht sehr verdünnte Lösung wie z. B. bei Chininsulfat Ursache der Verzögerung und des längeren Verharrens der Ausscheidung in Tropfenform ist, langsam und oft unvollstïndig beim Cinchonidin. Aus diesem Grunde zeigen die Cinchonidinsalze gewöhnlich neben sehr entwickelten und sehr complicirten Sternen, in der Entwickelung auf verschiedenen Abstufungen zuruickgebliebene Gebilde: z. B. glattrandige, nicht durchsichtige Tropfen, Tropfen mit behaurter oder stachliger Peripherie, halb bis ganz entwickelte einfachstrahlige, das licht nur schwach durchlassende, und darum bei durchfallendem Licht dunkel, bei auffallendem Lichte porcellanartig weiss erscheinende Sterne. 
Abgesehen hiervon ist ein wesentlicher Unterschied zwischen den Gestalten des Chinins und Cinchonidins nicht nachzuweisen.

Chinin.

Fig. 1. Vergrösserung: 110 fach. Fig. 2.
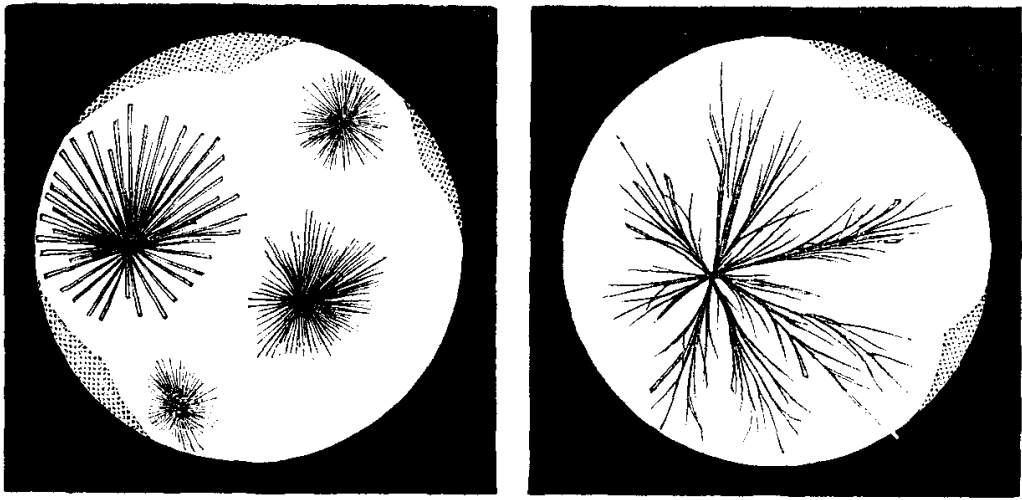

Cinchonidin.

Fig. 3.

Fig. 4.
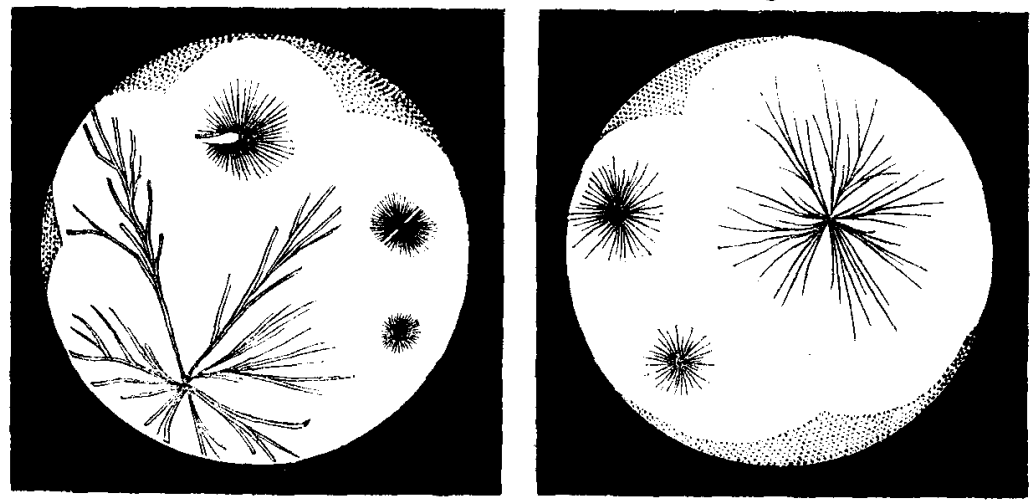

Das Cinchonin

zeigt einseitswendig, und darum in seiner primitivsten Gruppirung fächerartig, denselben Entwicklungsgang der Krystallgruppen wie das Chinin.

Die vorzugsweise von der Rhodanlösung aus in die Cinchoninlösung gleichsam hineinwachsenden Strahlen sind theils 
nadelförmig, theils flach, an der Spitze theils rechtwinklig, theils schräg abgeschnitten, von sehr ungleicher Länge und Derbheit, der Regel nach gerade, aber doch sehr häufig auch gebogen vorkommend, theils einfach bleibend, theils sich verästelnd.

Das Gesammtbild ist bei dünneren Strahlen einem Grasfelde (Fig. 6) sehr ähnlich, bei den derberen liesse sich ein Vergleich mit Equisetum (Fig. 7) rechtfertigen, ist aber nicht selten der Vergleich mit treppenartig gezackten Schiefergebilden (Fig. 5) zutreffender.

Cinchonin.

Fig. 5 .

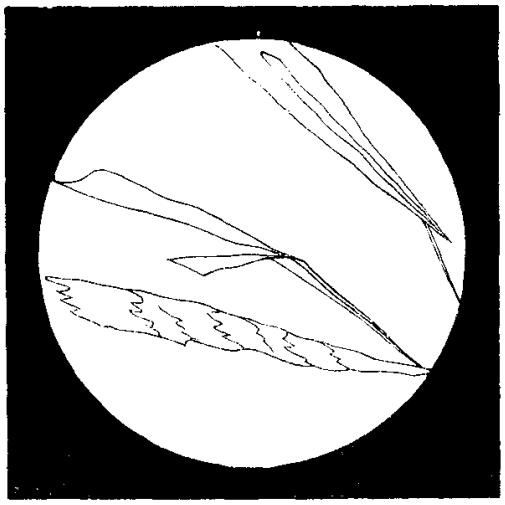

Fig. 7 .

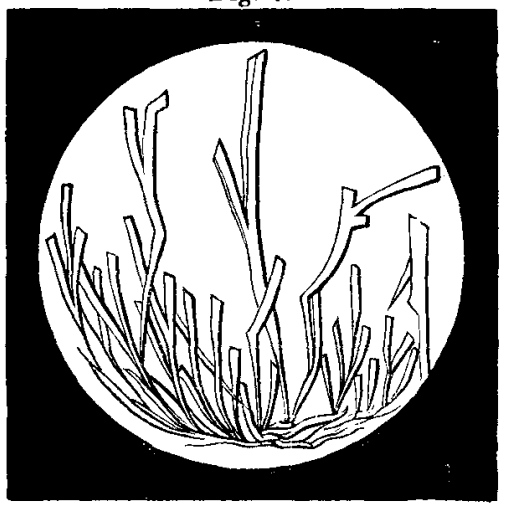

Fig. 6.

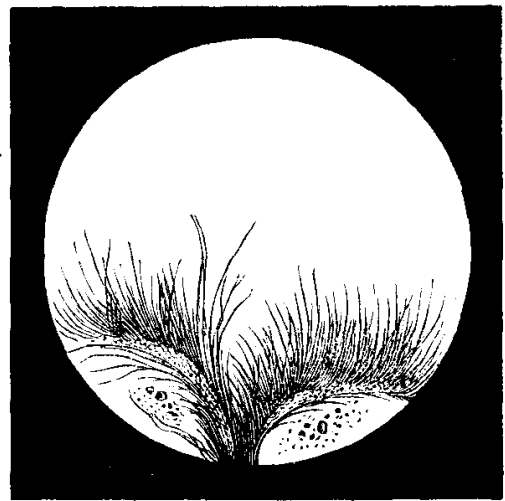

Das Chinidin

bildet bei seinem Zusammentreffen mit Rhodankaliumlösung zunächst eine sich ausscheidende halbdurchsichtige Flüssigkeit, welche theilweise tropfenartig in der umgebenden farblosen Flïssigkeit vertheilt ist.

Sowohl aus der cohärenten als auch aus der tropfenartig vertheilten Ansscheidung wachsen 
sehr rasch dendritische halbamorphe oder krystallinische Gruppirungen in die umgebende Flüssigkeit hinein, welche in ihren verschiedenen Formen gleichzeitig vorhanden sind, aber auch vereinzelt auftreten können. Sie sind bei durehfallendem Licht dunkel, bei auffallendem Licht porzellanartig, in beiden Fällen, soweit sie dendritischer Natur sind, wunderbar schöne Bilder darstellend.

Die dendritischen Figuren sind bald vergleichbar mit Zweigen von Thuja, (Fig. 10) bald mit Zweigen von Juniperus communis, (Fig. 9) zuweilen auch mit sterilen oder

\section{Chinidin. $\beta$ Kerner.}

Fig. 8.

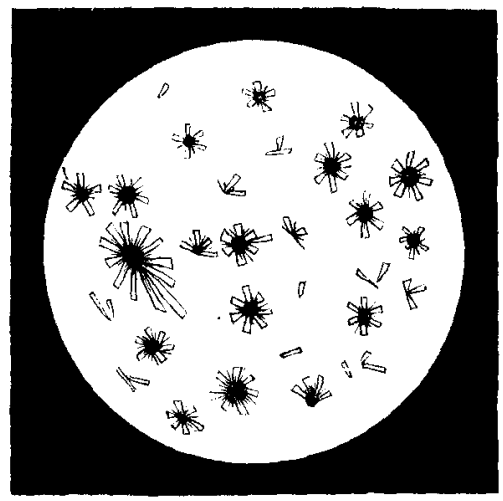

halb entblätterten Zweig'en dieser Pflanzen. Ausserdem kommen dicke tropfenartige undurchsichtige runde Formen vor, welche in ihrer Peripherie mehr roder weniger derbe kurze Strahlen zeigen (Fig. 8).

Bei den verschiedenen Beobachtungen ist bald die eine, bald die andere der vier genannten Formen vorherrschend.

Arch, d. Pharus. XIIt. Bds, 1. Heft.
Fig. 9.

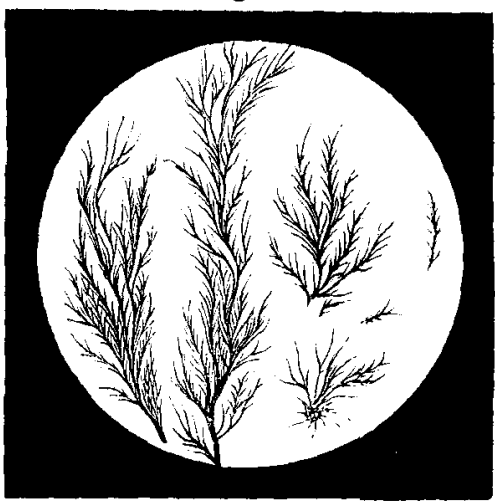

Fig. 10.

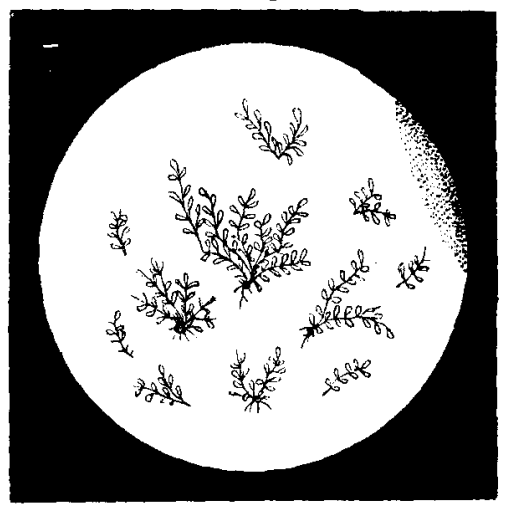

3 
Entscheidend sind nur die beiden ersten und darum Wiederholung der Versuche dann unerlässlich, wenn nur die beiden letztgenannten Formen oder eine derselben sich zeigen sollte.

Die Zartheit der beiden erstgenannten Formen bedingt ihre leichte Zerstörbarkeit; nicht vollständig wagerechte Lage des Objects, zu dicke Tropfen oder überhaupt jeder eine Bewegung des Objects bewirkende Umstand kann ibre Entstehung hindern.

Will man die mikroskopische Rhodanreaction anwenden auf Mischungen der Salze verschiedener Chinabasen, so empfiehlt es sich, Mischungen von bekannter Zusammensetzung: vorräthig zu haben und die entstehenden charakteristischen Bilder zu vergleichen.

Die Beabachtungsresultate sind recht gut in den Fällen, wo man es nur mit zwei Alkaloilden zu thun hat, weniger und jedenfalls nur bei nnmittelbarem Vergleich brauchbar, wenn mehr als zwei Alkaloïde gleichzeitig vorhanden sind.

Im Allgemeinen begegnet man bei diesen Versuchen häufig der Thatsache, dass Mischungen der Salze mehrerer Alkaloïde sich weniger rein krystallinisch, also leichter amorph oder halbamorph ausscheiden, als die ungemischten Salze, und aus dieser Abnahme der Krystallisationsfähigkeit resultirt die Entstehung mikroskopischer Gruppirungen, welche anf einfache Combination der aus den einzelnen Chinasalzen entstehenden Krystallgruppen nicht zurückgeführt werden können, wie folgende Versuche zeigen werden.

Chininsulfat wird am häufigsten Gegenstand der Prïfung auf Beimengung anderer Chinaalkoloïde sein, und dio Flüssigkeit, welche behufs Ausführung der obligatorischen Kerner'schen Chininprobe durch Mischung von 1 Theil käuflichen Chininsulfats mit 10 Theilen Wasser entsteht, ist für die mikroskopische Probe sehr passend und deshalb bei den hier erwähnten Versuchen vorausgesetzt.

Enthält das Chininsulfat 1\%-5\% Cinchonidinsulfat, so wird die Bildung der Sterne wesentlich verlangsamt, 
und es ist erst nach $1-3$ Stunden die Entwickelung von dichtstrahligen, theilweise unvollständig entwickelten Sternen und amorphen Tropfen mit oder ohne gleichsam borstenhaariger Peripherie beendet. Enthält das Chininsulfat 1\%-5\% Chinidin beigemengt, so zeigt sich die Bildung der Sterne zahlreicher als bei reinem Chininsulfat, mit Strahlen, welche meist einfach und sehr dicht gestellt sind. Ausserdem zeigen sich gelbe amorphe Trophen, theils isolirt, theils mehr oder weniger gruppirt zu Bildungen, welche nm so mehr der bei reinem Chinidin erwähnten Thujaform sich nähern, je grösser der Procentgehalt an Chinidinsulfat ist. Enthält das Chininsulfat $1 \%-5 \%$ Cinchoninsalz beigemengt, so tritt bei $1 \%$ Beimengung die Bildung von ziemlich vielen dichten Sternen ein, deren Strahlen einfach, nicht selten haarartig gebogen, zuweilen mit breiteren derben Strahlen gemischt sind.

Ein Gehalt von $2 \%-5 \%$ Cinchonin zeigt dieselben Formen, denen sich aber dendritenartige, den Laubmoosen vergleichbare Gebilde hinzugesellen, und zwar in dem Maasse mehr, als der Cinchoningehalt steigt.

Cinchoninsulfat, welches $1 \%$ Cinchonidinsulfat beigemengt enthält, zeigt mikroskopische Erscheinungen, welche von denen des reinen Cinchoninsulfats nicht wesentlich abweichen, obgleich im Ganzen die dünnstrahlige, oben als grasähnlich bezeichnete Form vorherrscht.

Eine Beimengung von $10 \%$ Cinchonidin bringt von der reinen Cinchoninform sehr abweichende dendritische Gebilde hervor, von denen einzelne allenfalls moosähnlich genannt werden könnten.

\section{Chinidinum sulfuricum venale}

besteht, abgesehen von relativ geringen Mengen anderer Chinaalkaloïde, welche seinen Verwendungswerth wohl kaum beeinflussen, im Wesentlichen aus den Sulfaten des Chinidins und Cinchonidins in sehr wechselnden relativen Verhältnissen, und zwar, soweit sie bei grossem Verbrauch und häufig 
wiederkehrenden Untersuchungen, mir vorkamen, in deu letzten Jahren ungleich mehr Cinchonidin enthaltend als früher.

Die Löslichkeit des Chinidinsulfats in Wasser (bei $10^{\circ}=$ $1: 108$ Hesse; bei $15^{0}=1: 98$ bis 110 Kerner) und des Cinchonidinsulfats (bei $12^{\circ}=1: 97,5$ Hesse; bei $15^{\circ}=1: 95$ bis 100 Kerner) ist annähernd $=1: 100$. Es dürfte demnaeh für die mikroskopische Prüfung des käuflichen Chinidinum sulfuricum ausreichen, dasselbe mit 100 bis 110 Theilen Wasser anzureiben oder zu schütteln, obgleich die Pharmacopoea Austriaca für Chinidinum sulfuricum eine Löslichkeit in Wasser von 1:100 bis $1: 300$ vindicirt. Möglichst vollständige Lösung des zu untersuchenden Fabrikates ist für unsern Zweck allerdings erforderlich, weil andernfalls nicht gerade nothwendig beide Sulfate im Verhältniss ihres Vorhandenseins sich lösen. Trockene Mischungen reiner Präparate, welche z. B. 10\%, 20\%, 30\% Chinidinsulfat enthielten, geben mit der 10 fachen Menge kalten Wassers angerieben, nach einigen Stunden annähernd ganz gleiche mikroskopische Bilder, ein Resultat, welches nicht auffällt, wenn man bedenkt, dass in allen 3 Fällen Chinidin bis zu seiner Löslichkeitsgrenze vorhanden war.

Eine Mischung ruiner Sulfate, vollständig in 100 Theilen Wasser gelöst, gab bei einem Chinidingehalt von $10 \%$ keine dendritische Bildung, dagegen nach mehreren Stunden wenige dichtstrahlige Sterne und Tropfen mit rauher Peripherie; von $15 \%$ : dieselben Formen, aber zahlreicher und vollständiger entwickelt; von 20\%: dieselben Formen, theilweise zu dendritischen Gebilden sich vereinigend; von $25 \%$ : wie bei $20 \%$, aber mit etwas stärker entwickelten Formen, auch erst nach mehreren Stuuden; von $33 \%$ Chinidingehalt: schon nach 30 Minuten dendritische Bildungen, welche Fucus vergleichbar sind, nach mehreren Stunden aber die einzelnen Strahlen fächerartig krystallinisch entwickeln; bei $50 \%$ und mehr: nach wenigen Minuten dendritische Gebilde, welche im Ganzen den bei reinem Chinidin als thrjaähnlich bezeichneten Formen gleichen. - Erst nach mehreren $(5-10)$ Stun- 
den erscheinen dann nachträglich sehr scharf ausgeprägto und gut ausgebildete Sterne neben und zwischen jenen dendritischen Formen.

\section{Ueber Chloroform.}

Von Ir. G. Vulpius in Heidelberg.

Es ist in den letzten Zeiten so viel über Chloroform im Allgemeinen und uber Chloralchloroform im Besonderen in der pharmaceutischen Presse gesprochen worden, ohne dass die Acten über diesen Gegenstand geschlossen erscheinen, und so mag es denn gestattet sein, hier einige Erfahrungen aus dem Operationssaal einer grossen chirurgischen Klinik mitzutheilen, wobei jedoch absichtlich keine Bezugsquelle genannt werden soll, um von vornherein jede Möglichkeit eines Gedankens an indirekte Empfehlung irgend einer Firma auszuschliessen.

Da das gewöhnlich benutzte Chloroform etwa vor Jahresfrist einigemale zu wïnschen übrig gelassen hatte, so wurden sechs verschiedene Chloroformsorten aus diversen Fabriken bezogen nnd zwar 3 mal gewöhnliches, 2 mal ans Chloral dargestelltes und 1 mal englisches Patentchloroform. Jede Chloroformsorte erhielt ihre Nummer und wurde unter dieser ohne jede weitere Bezeichnung der Provenienz oder des Preises in den Operationssaal geliefert. Als nach Monatsfrist zwei Sorten als vorziiglich, eine als sehr mangelhaft in der Wirkung bezeichnet worden war, so wurden die Nummern gewechselt und auch jetzt waren die vorher als vortrefflich bezeichneten Chloroformsorten alsbald wieder unter ihren neuen Nummern lieraus gefunden. Es befand sich aber unter diesen kein Chloroform aus Chloral, auch war es nicht das englische, sondern gewöhnliches Chloroform, welches jedoch die rigorose Probe des Stehenlassens mit concentrirter Schwefelsäure vollkommen ohne die geringste Färbung ausgehalten hatte. Dass man unter solchen Umständen von Anschaffung des theueren Chloral-Chloroforms doppelt gern 